\section{Interaction of Arylthioureas and Aromatic Sulphonyl Chlorides}

IT was recently shown ${ }^{1}$ that the reaction of arylureas and aromatic sulphonyl chlorides in pyridine under restrained conditions does not yield arylsulphonyl arylureas but occurs with simultaneous dehydration, and results in the formation of arylsulphonyl arylcyanamides in good yields. The production of the appropriate substituted melamines was also observed in $\operatorname{som} \theta$ cases $^{2}$. In continuation of this work, the interaction of sulphonyl chlorides and arylthioureas has been examined.

The action of benzenesulphonyl chloride on thiourea in alcoholie solution was first studied by Remsen and Turner ${ }^{3}$, who obtained dithioformamidine dihydrochloride and diphenyl disulphoxide; similar results were obtained by Leitch et $a .^{4}{ }^{4}$ using $p$-acetamido. benzenesulphonyl chloride. Fromm and Heyder's attempts ${ }^{5}$ to prepare the corresponding aryl-substituted dithioformamidine by the action of sulphonyl chlorides on phenylthiourea resulted in ring-closure, with formation of $2: 4$-diphenyl-3:5 di-imino-2:3 : $4: 5$-tetrahydro-1 : $2: 4$-thiadiazole.

In the present investigation, using conditions recently employed with arylureas, the interaction of arylthiouroas and aromatic sulphonyl chlorides was found to take a different course. The aromatic thiourea is rapidly desulphurized giving arylcyanamide as one of the main products, and the reaction then proceeds essentially as with arylureas. ${ }^{1}$ Thus, treatment of 1 mole of arylthiourea with 2.5 moles of sulphonyl chloride in pyridine at moderate temperatures gives a product from which approximately 1 gm. molecule each of arylcyanamide (I) and sulphur are obtained. Some of the cyanamide reacts further with the excess of sulphonyl chloride, as shown by the isolation of arylsulphonyl arylcyanamide (II). Part of the sulphonyl chloride is converted to the disulphoxide (III) during the reaction.

Disregarding, for the time being, the mechanism of the reaction, the net results can be represented by the following overall equation :

\section{Structure of the Diazonium Ion}

AcCordING to the accepted constitution of the diazonium ion, a nitrogen atom takes the place of three hydrogen atoms in a substituted ammonium ion. It is accordingly conceivable that there may be resonance between the two forms :

$$
\left[\begin{array}{ll}
R & \mathrm{~N} \\
& \mathrm{~N} \\
& \mathrm{~N}
\end{array}\right]^{+}
$$$$
\left[\begin{array}{ll} 
& \mathrm{N} \\
R & \mathrm{III} \\
& \mathrm{N}
\end{array}\right]^{+}
$$

By labelling one of the nitrogen atoms, we have shown that such resonance does not, in fact, occur.

Starting with phthalimide containing labelled nitrogen, we synthesized and diazotized labelled aniline and coupled it with $\beta$-naphthol. By reduction we split the product to aniline and $\alpha$-amino- $\beta$-naphthol. If resonance of the above kind exists, the labelled nitrogen should be equally divided between the aniline and the amino-naphthol. If, on the other hand, such resonance is absent, the labelled nitrogen should be entirely in the aniline. According to our preliminary results, more than 97 per cent of the labelled nitrogen remains in the aniline. We conclude that there is no resonance.

We are indebted to the Mass Spectrometer Group, Atomic Energy Research Establishment, Harwell, who assayed our nitrogen samples.

$$
\begin{aligned}
& \text { P. F. HoLT } \\
& \text { BetTy I. BULLock }
\end{aligned}
$$

Department of Chemistry,

University of Reading. Jan. 19.

\section{The Reaction between $\beta$-Carotene and Antimony Trichloride}

IT has always seomed anomalous that anhydrovitamin A with six conjugated double bonds should give $\lambda_{\max }$ at $620 \mathrm{~m} \mu$ with the Carr-Price reagent, and that $\beta$-carotene with eleven conjugated double

$$
\begin{aligned}
& 3 R \cdot \mathrm{NH} \cdot \mathrm{C}: \mathrm{NH}+3 R^{\prime} \cdot \mathrm{SO}_{2} \mathrm{Cl} \longrightarrow 3 R \cdot \mathrm{NH} \cdot \mathrm{CN}+3 \mathrm{~S}+R^{\prime} \cdot \mathrm{SO} \cdot \mathrm{SO} \cdot R^{\prime}+R^{\prime} \cdot \mathrm{SO}_{2} \mathrm{OH}+3 \mathrm{HCl}+\mathrm{H}_{2} \mathrm{O} \\
& \text { SH } \\
& \overbrace{R^{\prime} \cdot \mathrm{SO}_{2} \cdot \mathrm{N} R \cdot \mathrm{CN}}^{R^{\prime} \mathrm{SO}_{8} \mathrm{Cl}} \\
& \text { (III) } \\
& \text { (II) }
\end{aligned}
$$

As an example, the interaction between phenylthiourea and toluene-p-sulphonyl chloride gave phenylcyanamide (65 per cent yield), sulphur (80 per cent, based on the thiourea), toluene-p-sulphonyl phenylcyanamide (25 per cent) and di-p-toluene disulphoxide (25 per cent). Similar results are obtainable with other arylthioureas.

Further experiments in connexion with this work are in progress, and a detailed account will appear elsewhere in due course.

\section{Frederick Kurzer}

Royal Free Hospital School of Medicine, University of London. Jan. 5.

${ }^{1}$ Kurzer, J. Chem. Soc., 1034, 3029 (1949).

${ }^{2}$ Kurzer, J. Chem. Soc., 3033 (1949)

${ }^{3}$ Remsen and Turner, Amer. Chem. J., 25, 190 (1901).

* Leitch, Baker and Brickman, Canad. J. Res., 23 B, 139 (1945).

${ }^{5}$ Fromm and Heyder, Berichte, 42, 3804 (1909). bonds should give $\lambda_{\max }$ at $585 \mathrm{~m} \mu$. Further, $\varepsilon_{\max }$ for anhydrovitamin $A$ is 170,000 , whereas that for $\beta$ carotene at $585 \mathrm{~m} \mu$ is only $22,000^{1}$.

Assuming that the compounds formed in the reaction are unsymmetrical ions, $\lambda_{\max }$ should be a linear function of the number of conjugated double bonds 2 . In the colour test, anhydrovitamin $A_{1}$ (with six conjugated double bonds) has $\lambda_{\max }=623 \mathrm{~m} \mu^{3}$, whereas anhydrovitamin $\mathbf{A}_{2}$ (with probably seven conjugated double bonds) has $\lambda_{\max }=693 \mathrm{~m}^{4}$. Hence for eleven conjugated double bonds one would expect $\lambda_{\max }=693+4(693-623)=973 \mathrm{~m} \mu$. Also, axerophthene (five conjugated double bonds) has $\lambda_{\max }=$ $577 \mathrm{~m} \mu^{5}$ in the colour test and iso-desmethylaxerophthene (four conjugated double bonds) has $\lambda$ max $=$ $490 \mathrm{~m} \mu^{8}$. Hence for eleven conjugated double bonds one would expect $\lambda_{\max }=577+6(577-490)=1,099$ $\mathrm{m} \mu$. The assumptions here debar quantitative prediction, but they prompt investigation in the region 